\title{
Conocimiento ecológico tradicional y conductas hacia la ecoeficiencia agrícola en pobladores de la provincia de Junín, Perú
}

Traditional ecological knowledge and behaviors towards agricultural ecoefficiency in people of the province of Junín, Peru

\author{
${ }^{1, a}$ Roberto I. Beltrán Palomares \\ ${ }^{2, \mathrm{~b}}$ Marino Ninalaya Casallo \\ ${ }^{3, c}$ Herbert Víctor Huaranga Rivera \\ ${ }^{4, \mathrm{~d}}$ Edith Karina Valero Misari \\ ${ }^{5, \mathrm{e}}$ Lucy Reyes Poma
}

\begin{abstract}
ARTÍCULO ORIGINAL
${ }^{1}$ Universidad Nacional del Centro del Perú. Facultad de Ingeniería y Ciencias Humanas. Huancayo, Perú E-mail: rbeltran71@hotmail.com. ${ }^{2}$ Universidad Nacional Intercultural de la Selva Central Juan Santos Atahualpa. Junín, Perú. E-mail: mninalayac@gmail.com.

${ }^{3}$ Universidad Nacional Intercultural de la Selva Central Juan Santos Atahualpa. Junín, Perú. E-mail: huancayo huaranga@hotmail.com. ${ }^{4}$ Universidad Nacional Intercultural de la Selva Central Juan Santos Atahualpa. Junín, Perú. E-mail: paravariar.doc@gmail.com ${ }^{5}$ Ministerio de Educación. Lima, Perú.E-mail: lucyreipol@gmail.com.

aORCID: 0000-0002-2482-1925

bORCID: 0000-0002-8684-150X

'ORCID: 0000-0002-8054-4213

${ }^{\mathrm{d} O R C I D}$ 0000-0001-9290-4522

'ORCID: 0000-0002-6963-8147
\end{abstract}

Palabras clave: Conocimiento ecológico tradicional, ecoeficiencia, ecoeficiencia agrícola, Junín.

Keywords: Traditional ecological knowledge, ecoefficiency, agricultural ecoefficiency, Junín

\section{Información adicional}

Presentado: 30/09/2020

Aprobado: 14/12/2020

\section{RESUMEN}

La industrialización de la agricultura ha generado una producción a gran escala y en menor tiempo, originando daños ambientales, en muchos casos irreversibles. En este proceso se han utilizados diferentes insumos químicos y bioquímicos que han empobrecido la tierra, contaminado los productos agrícolas y afectando incluso la salud humana. Esta situación amerita una atención inmediata de los diferentes sectores de la sociedad, con el soporte del conocimiento científico. La investigación se enmarcó dentro de un enfoque intercultural, interdisciplinario y transversal teniendo como soporte la filosofía del desarrollo sostenible. El objetivo fue determinar la relación que existe entre el conocimiento ecológico tradicional y las conductas hacia la ecoeficiencia agrícola en pobladores de la provincia de Junín, Perú. Se formuló la hipótesis: existe una relación significativa entre el conocimiento ecológico tradicional y conductas hacia la ecoeficiencia agrícola en los pobladores de la provincia de Junín. La investigación fue de tipo básica, nivel descriptivo y diseño correlacional. Se utilizó la técnica de la encuesta, y como instrumentos los cuestionarios de conocimientos ecológicos tradicionales y de prácticas de ecoeficiencia agrícola, diseñadas y validadas por juicio de expertos y por el coeficiente de Spearman-Brown. La población estuvo conformada por pobladores de las 24 comunidades de la provincia de Junín, Perú. La muestra estuvo constituida por 210 sujetos, 60 damas y 150 varones. Los resultados obtenidos a partir del análisis estadístico evidencian una relación significativa entre el conocimiento ecológico tradicional y las conductas hacia la ecoeficiencia agrícola en pobladores de Junín, con 0.05 de significancia y $\tau=0.587$ y $Z_{t}(1.87)<Z_{c}(5.87)$.

\section{ABSTRACT}

The industrialization of agriculture has generated large-scale production in a shorter time, causing environmental damage, in many cases irreversible. In this process, different chemical and biochemical inputs have been used that have impoverished the land, contaminated agricultural products and even affected human health. This situation deserves immediate attention from different sectors of society, with the support of scientific knowledge. The research was framed within an intercultural, interdisciplinary and transversal approach, having as support the philosophy of sustainable development. The objective was to determine the relationship between traditional ecological knowledge and behaviors towards agricultural ecoefficiency in residents of Junín Province, Peru. The hypothesis was formulated: there is a significant relationship between traditional ecological knowledge and behaviors towards agricultural ecoefficiency in the inhabitants of Junín Province. The research was of basic type, descriptive level and correlational design. The survey technique was used, and as instruments the questionnaires of traditional ecological knowledge and agricultural ecoefficiency practices, designed and validated by expert judgement and the Spearman-Brown coefficient. The population was made up of people from the 24 communities in the province of Junín, Peru. The sample was made up of 210 subjects, 60 ladies and $150 \mathrm{men}$. The results obtained from the statistical analysis show a significant relationship between traditional ecological knowledge and behaviors towards agricultural ecoefficiency in Junín's inhabitants, with 0.05 of significance. and $\tau=0.587$ and $\mathrm{Zt}(1.87)<\mathrm{Zc}$ (5.87). 


\section{INTRODUCCIÓN}

El conocimiento ecológico tradicional constituye un cuerpo de conocimientos, prácticas y creencias en base a una determinada cosmovisión, transmitida de generación en generación, a través de diversas formas culturales con el propósito de difundir, proteger y mantenerla viva a lo largo de la historia. Investigadores argumentan a favor de las bondades de los conocimientos tradicionales sobre la gestión agrícola a través del uso racional y eficiente de los recursos en los procesos productivos.

La necesidad de incrementar la productividad, en menor tiempo y a gran escala, ha agudizado la contaminación ambiental, quebrando la armonía de los diferentes ecosistemas y empobrecido la tierra agrícola (Barcia-Piedras, 2020). El uso de fertilizantes y otros elementos químicos generan cada vez más dependencia en el sector productivo, también contaminación y enfermedades (Alpuche, 2019). Esta problemática requiere de una atención impostergable (Ikeda, 2020).

Para paliar el impacto de las técnicas actuales en la labor agrícola, se han revisado múltiples propuestas y técnicas, encontrando como una de las más viables y coherentes, la integración del conocimiento tradicional con el conocimiento científico (Gilles \& Yucra, 2019). El conocimiento tradicional sobre el manejo y producción en las tierras agrícolas no daña ni al ecosistema ni al ambiente, además, también puede lograr producciones agrícolas exitosas (De la Riva, 2019).

El conocimiento ecológico tradicional tiene como esencia el bagaje de saberes milenarios, prácticas y creencias que mantienen en permanente unión y equilibrio a los seres humanos con el ambiente (Fonseca, 2016). Integrar armónicamente hombre - naturaleza, a partir de la comprensión de los mensajes subliminales de los mitos, cosmovisiones y rituales que explican el origen de la tierra y su respectivo aprovechamiento de manera sostenible, constituye una posibilidad manejable y accesible. Evitar que estos conocimientos sean reemplazados por la nueva "tecnología" impediría su nefasto impacto en la diversidad biológica de los diferentes sitios silvestres, así como en los agroecosistemas saludables, logrando conservar lo que aún queda y a partir de allí remontarlo (Arnaiz-Schmitz, 2018).

Por otro lado, la forma de cubrir las necesidades alimentarias ha cambiado de una dinámica de autoabastecimiento a una dinámica de mercado, que arroja resultados ambivalentes (Shackleton et al., 2015), conllevando ineludiblemente a tener resultados negativos para la viabilidad y sostenibilidad de los recursos, en una constante promoción del consumismo y depredación (Lima - Da Silva et al., 2019). Esta manera de "consumismo" genera deforestación, cambio climático, pérdida de la biodiversidad, crisis del agua y desertificación de suelos. Entonces, revalorar, rescatar y aplicar los conocimientos tradicionales como tecnología no contaminante, conservacionista y ecoeficiente en el uso de los recursos naturales permitiría la subsistencia humana y ambiental. Los problemas de contaminación y perturbación de los ecosistemas (biósfera - organismos) son un producto histórico del mal manejo de los recursos del planeta, consecuencia de una deficiente administración de los insumos industriales, de la pobre concepción de los contaminantes, impulsada por la avaricia de un grupo de poder (Bolaños, 2016).

La sustentabilidad agroecológica heredada de las prácticas productivas ancestrales parten de la dualidad entre el ser humano y la naturaleza, relación e intercambio que debe albergar un principio simbiótico de aprovechamiento sin causar daños, creando una red viva de relaciones entre sí, una nueva cultura amigable con el ambiente que mejore el futuro de la humanidad (Castillo \& Venegas, 2016). Forjar una nueva educación ambiental dentro de la praxis intercultural y ancestral es el camino abierto para conservar especies y con ellas, la humanidad. Cuando se habla de sostenibilidad o desarrollo sostenible solo se piensa en disminuir el impacto negativo de los seres humanos en el mundo, pero concentrar energías en el desarrollo regenerativo es potenciar el impacto positivo del hombre en la Tierra. Aprender de los procesos 
regenerativos de la naturaleza puede crear un nuevo imaginario colectivo, centrando al biocentrismo como el camino para lograr el tan ansiado equilibrio entre el hombre y la naturaleza (Ruano et al., 2020)

En este sentido, Alvarez (2017) y Rodríguez (2018) coinciden en que el conocimiento ecológico tradicional conducen a la ecoeficiencia agrícola, pues ofrece una amplia gama de posibilidades para el desarrollo armónico hombre - naturaleza, asimismo, una gran flexibilidad para adaptarse a las nuevas situaciones que se originan del contexto actual, lo que llevará inevitablemente a la ecoeficiencia agrícola que cubra las necesidades alimentarias y de recursos naturales de los diferentes pueblos.

Entender la ecoeficiencia agrícola como la capacidad y calidad de la tierra para ser económicamente rentable y ambientalmente sostenible (Riechmann, 1995; Achkar et al., 2005; Twarog \& Promila, 2004) genera mejores posibilidades de encontrar soluciones adecuadas a las necesidades de los habitantes de cualquier lugar. La finalidad de la ecoeficiencia agrícola es encontrar un uso óptimo y responsable de recursos, mejorando la productividad, contribuyendo a la satisfacción de necesidades de manera equitativa y sostenible, reduciendo la huella ecológica negativa que deja el hombre para generaciones venideras. En el mismo ámbito, se pueden encontrar tres dimensiones: ambiental, que busca preservar y potenciar la diversidad y complejidad de los ecosistemas, su productividad, los ciclos naturales y la biodiversidad, siendo sostenible a través del tiempo (Riechmann, 1995); social, que prioriza el acceso equitativo a los bienes de la naturaleza, tanto en términos intergeneracionales como entre géneros y entre culturas, entre grupos y clases sociales y también a escala del individuo (Achkar et al., 2005); económico, en referencia a todas las actividades humanas relacionadas con la producción, distribución, consumo de bienes y servicios en la producción agrícola.

Por otro lado, el conocimiento ecológico tradicional o prácticas ambientales basadas en la etnoecología y conocimiento tradicional de las comunidades originarias de la provincia de Junín cuenta con conocimientos ancestrales y culturales que le permite obtener el mejor aprovechamiento del ambiente, desarrollado dentro de prácticas comunitarias, instituciones, relaciones sociales y rituales ( Twarog \& Promila, 2004).

Las investigaciones realizadas en estos campos coinciden en que a través de las políticas de negociaciones e intervenciones sostenibles sobre la tierra en base a los conocimientos ancestrales se establecen culturas amigables y conservacionistas sobre los procesos de producción agrícola. Las prácticas agrícolas con fundamento originario ancestral plantean un importante reto ontológico a la política consumista y expansionista para desarrollar negociaciones y prácticas ecoeficientes (Vargas, 2011). También, el conocimiento ecológico tradicional puede ser de interés a la población a través de la producción forestal que aporta económicamente a las familias campesinas. Además, su aplicación puede ser útil para recuperar la fertilidad de suelos degradados, promover la conectividad, mejorar la salud del ecosistema selvático y enfrentar problemas de escasez de productos forestales básicos, como leña y material (Levy et al., 2015), además, que los conocimientos ecológico tradicionales junto con las creencias y prácticas influyan en los valores doméstico, económico, medicinal, ritual y ontológico otorgados a la flora y fauna de sus bosques; asimismo, en el correcto uso de las tierras de cultivo (Millán-Rojas et al., 2016).

Considerando lo anterior, el problema de investigación fue ¿cuál es la relación que existe entre conocimiento ecológico tradicional y conductas hacia la ecoeficiencia agrícola en pobladores de la provincia de Junín? Para ello, se sistematizaron los saberes ecológicos tradicionales y se determinó la relación con las actitudes en ecoeficiencia agrícola. El objetivo fue determinar la relación que existe entre conocimiento ecológico tradicional y conductas hacia la ecoeficiencia agrícola en pobladores de la provincia de Junín, Perú. La hipótesis planteada fue que existe una relación significativa entre el conocimiento ecológico tradicional y conductas hacia la ecoeficiencia agrícola en los pobladores de la provincia de Junín. 


\section{MATERIAL Y MÉTODOS}

La población estuvo conformada por los pobladores de las 24 comunidades quechuas de la provincia de Junín, Perú. Estas comunidades fueron: Carhuamayo, Huanchuyro, Huayre, Jachahuanca, Mariano Nieves Chavez, Matacancha, Pampallacta, Pancha Grande, Piscurruray, Quilcatacta, Quipacancha, Quishuar, Rayannioc, San Alejandro De Yuraj Ojcha, San Juan De Hornomachay, San Juan de Shalacancha, San Miguel de Puyay, San Pedro de Pari, San Pedro De Shacpan, Santa Rosa de Tama, Tambos, Ulcumayo, Villa de Junín y Yapacmarcaque, que habitan aproximadamente sobre los $4000 \mathrm{~m} \mathrm{~s}$. n. m. y tienen como población identificada como quechua a 338 pobladores (BDPI, 2020).

La muestra fue elegida por muestreo no probabilístico, determinada por accesibilidad de los participantes. Se logró encuestar a 210 personas, de las cuales 60 fueron damas y 150 fueron varones residentes en la zona de estudio, a quienes se encuestó con los instrumentos de conocimiento ecológico tradicional y conductas hacia la ecoeficiencia agrícola. El test fue aplicado inicialmente como prototipo y se validó por juicio de expertos antes de su aplicación. Las evaluaciones abarcaron las dos variables de estudio V1: conocimiento ecológico tradicional y V2: conductas hacia la ecoeficiencia agrícola. Las dimensiones correspondientes a cada variable se exponen en la Tabla 1.

\section{Tratamiento estadístico}

Se determinaron los puntajes efectivos de los rangos para cada variable de estudio, incluyendo sus dimensiones, determinándose una correlación entre las variables a través de la prueba estadística de correlación Tau b Kendall (fórmula 1)

$$
\tau(\text { tau })=\frac{S}{\frac{1}{2} N(N-1)}
$$

Dónde: $($ tau $)=$ coeficiente de correlación de Kendall, $\mathrm{S}=$ puntuación efectiva de los rangos.

Tabla 1

Matriz de dimensiones relativas a las variables de estudio

Variables

\begin{tabular}{l|l}
\hline $\begin{array}{l}\text { Variable 1: } \\
\text { Conocimiento } \\
\text { ecológico } \\
\text { tradicional }\end{array}$ & Conocimientos tradicionales del agua. \\
\cline { 2 - 2 } & Conocimientos tradicionales del suelo y del medio natural. \\
\cline { 2 - 2 } & Conocimientos tradicionales sobre abonado de la tierra. \\
\cline { 2 - 2 } $\begin{array}{l}\text { Variable 2: } \\
\text { Conductas hacia } \\
\text { la ecoeficiencia }\end{array}$ & Conocimientos tradicionales sobre tratamientos de plagas. \\
\cline { 2 - 2 } \begin{tabular}{l} 
agrícola \\
\cline { 2 - 2 }
\end{tabular} & Conductas hacia la ecoeficiencia en la gestión del agua. \\
\cline { 2 - 2 } & Conductas hacia la ecoeficiencia en la conservación de suelos y del medio natural. \\
\hline & Conductas hacia la ecoeficiencia en el tratamiento de plagas. \\
\hline & Conductas hacia la ecoeficiencia en la gestión de residuos agrícolas. \\
\hline
\end{tabular}

Los rangos que se utilizaron para medir los resultados obtenidos acerca del conocimiento ecológico tradicional y las conductas hacia la ecoeficiencia agrícola son: 
Tabla 2

Baremo

\begin{tabular}{|l|l|}
\hline Valoración & Rango \\
\hline Alto & {$[100-75]$} \\
\hline Medio & {$[74-50]$} \\
\hline Bajo & {$[49-25]$} \\
\hline Muy bajo & {$[24-0]$} \\
\hline
\end{tabular}

Nota: Elaboración propia.

\section{RESULTADOS}

El puntaje más alto alcanzado se dio en relación al abonado de la tierra (83.40 pts.), evidenciando el uso actual de técnicas antiguas para el enriquecimiento de nutrientes en el suelo de cultivo. Los datos encontrados reportan que el proceso de abonado es donde se aplica con mayor énfasis los conocimientos ancestrales con la finalidad de conservar la estructura y textura del suelo de producción. El residuo agrícola en muy poca cantidad es aprovechado para la alimentación animal, quedando otra parte como potenciales agentes de contaminación. Sin embargo, a través de las técnicas ancestrales, los agricultores los convierten en abono orgánico, aprovechándolo de manera eficiente para mejorar los nutrientes del suelo.

En contraste, el puntaje más bajo lo alcanzó el tratamiento de plagas (61.23 pts.). Esto es un indicador de la dificultad que tienen los pobladores, en especial agricultores, para combatir plagas y enfermedades relacionadas en sus cultivos. Los tratamientos ineficaces en este campo pueden generar pérdidas económicas considerables. Entre las principales plagas que afectan los cultivos están el pulgón o mosca saltona, gorgojo de los andes, la rancha, verrugas causadas por hongos. Más del $50 \%$ de la muestra encuestada no pueden controlar las plagas con los conocimientos ecológicos tradicionales, en consecuencia, tienen que recurrir a fertilizantes sintéticos. Los pobladores son conscientes de los efectos que produce el uso excesivo de fertilizantes en el control de plagas como la modificación del pH del suelo, extinción de microrganismos favorables para la producción, la acidez de los suelos y la infertilidad de los mismos, perjudicial para la producción y la economía familiar. Sin embargo, la falta de efectividad de las técnicas ancestrales hace necesaria y obligatoria que los pobladores recurran al uso de los agroquímicos.

En relación al conocimiento del agua, suelo y gestión de residuos, los puntajes promedio fluctuaron alrededor de 75 pts., indicando un nivel aceptable en estas dimensiones. En ese sentido, se puede afirmar que existen buenas prácticas llevadas a cabo por los pobladores en gestión de los recursos que emplean durante la preparación del terreno, siembra, riego y cosecha de productos; además, el manejo tradicional de los residuos producto de la siembra y cosecha también son correctamente manejados, principalmente reinsertados a la cadena trófica como material de abono. Los residuos agrícolas son aprovechados de distintas formas y con criterio ecológico, la técnica más usada es depositar sobre el suelo y dejar que cumpla el ciclo de descomposición-humificación-mineralización, es decir, su conversión en humus y luego devolverla como materia orgánica al suelo.

Por otro lado, se muestran los resultados obtenidos de la aplicación de los instrumentos de recolección de datos de la variable "conductas hacia la ecoeficiencia agrícola" incluyendo las cinco dimensiones (conductas hacia la ecoeficiencia en la gestión del agua, conductas hacia la ecoeficiencia en la conservación de suelos y del medio natural, conductas hacia la ecoeficiencia en el abonado de la tierra, conductas hacia la ecoeficiencia en el tratamiento de plagas y conductas hacia la ecoeficiencia en la gestión de residuos agrícolas) (Figura 1). 


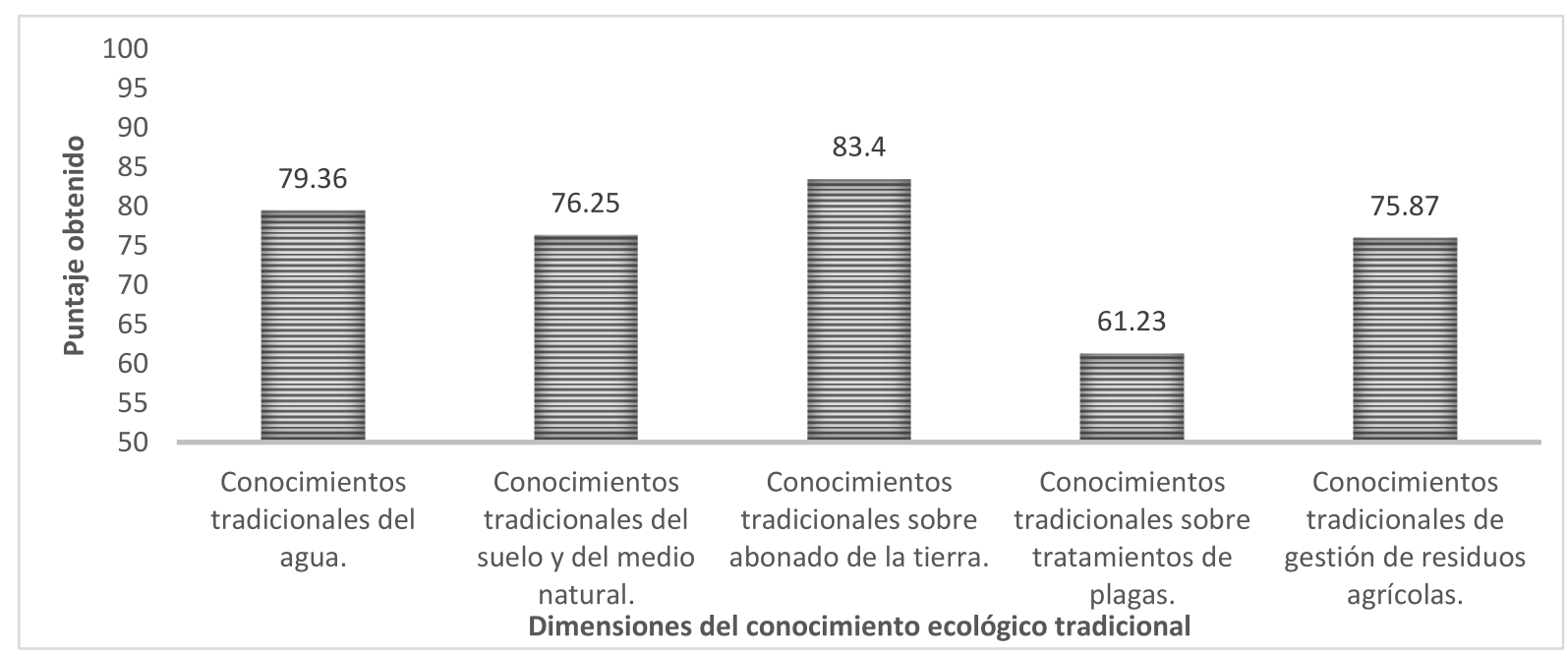

Figura 1. Puntajes obtenidos en cada dimensión de conocimiento ecológico tradicional

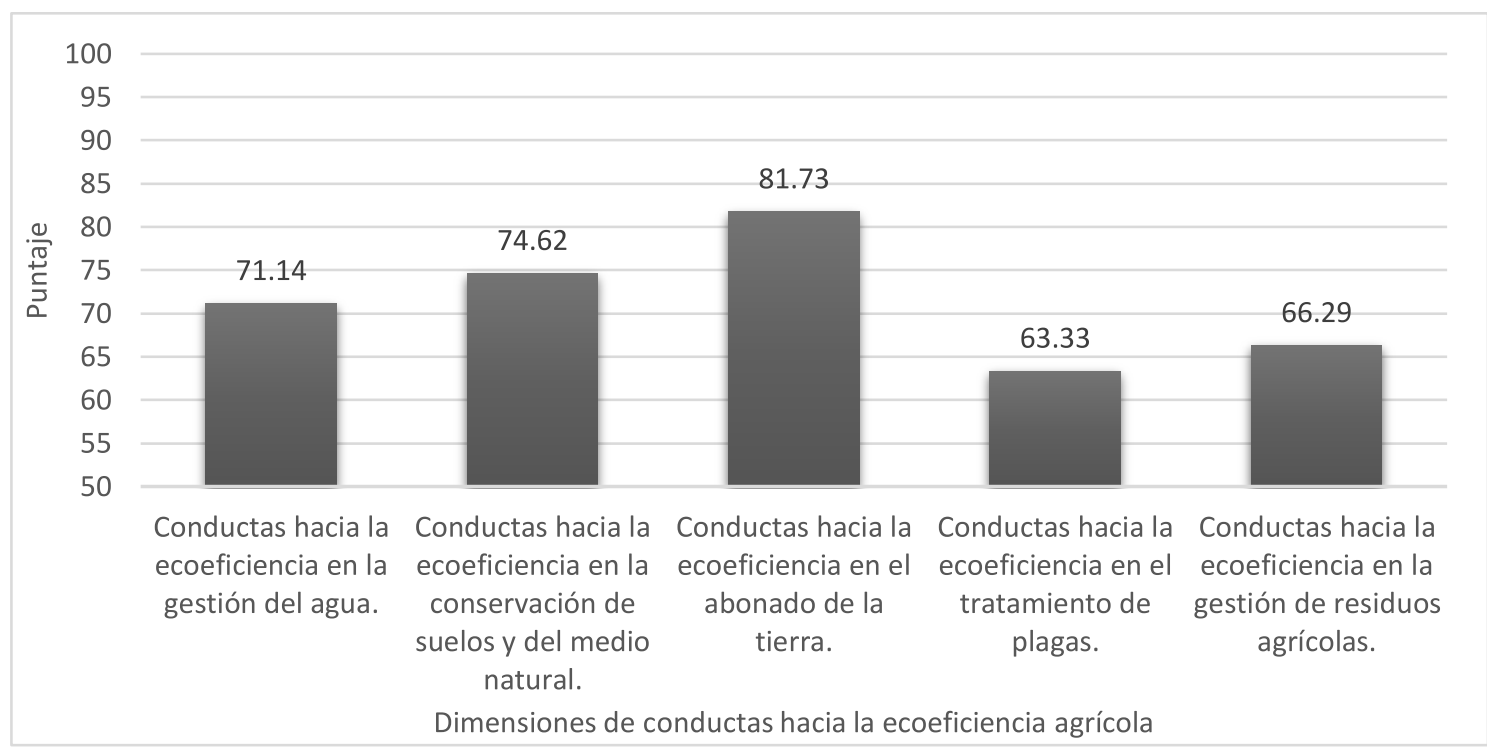

Figura 2. Puntajes obtenidos en cada dimensión de conductas hacia la ecoeficiencia agrícola

Las conductas ecoeficientes en el tratamiento de plagas son las que menos se aplican (63.33 pts.), esto como reflejo del poco conocimiento de técnicas eficaces para el control de elementos dañinos para los cultivos; la falta de conocimiento y el uso de técnicas poco eficientes conlleva a pérdidas considerables en la siembra, riego y cosecha de especies vegetales.

Por otro lado, la conducta mejor calificada es al abonamiento de la tierra ( 81.73 pts.) como resultado de la aplicación de técnicas eficaces para el enriquecimiento del suelo de siembra y favorecimiento del crecimiento de las plantas.

Por su parte, los puntajes relativos a gestión del agua y conservación de suelos no presentan una fluctuación apreciable en relación al conocimiento tradicional. Sin embargo, existió un claro descenso en cuestión de gestión de residuos agrícolas (66.29 pts.), esto como resultado de la poca capacidad efectiva para el manejo de los restos procedentes de la agricultura, aunque poseen conocimiento sobre cómo hacerlo (Figura 2).

Es importante recalcar que el conocimiento ecológico ancestral sabe que nutrir a la tierra y conservarla es importante, y para lograrlo es accesible y rentable utilizar los residuos agrícolas; siendo los restos de las 
leguminosas, gramíneas y crucíferas, las que más salud brindan al suelo. Sin embargo, en la actualidad es más lógico que solo se coseche lo sembrado, olvidando la importancia del abono verde.

Los resultados presentados anteriormente en la Figura 1 y Figura 2 indican que los pobladores de Junín poseen notable conocimiento tradicional y los aplican de forma eficiente durante el periodo de abono, preparación de suelo y siembra. Los periodos de riego y mantenimiento de los cultivos son llevados controladamente, sin causar mayor impacto sobre el ambiente. Sin embargo, al finalizar el ciclo agrícola, los pobladores no poseen conocimiento tradicional ni poseen actitudes de conservación ecológica suficientes para preservar el ambiente; y si se considera la baja actitud que presentan en este campo, a pesar del conocimiento que poseen, es probable que esto se deba a la falta de recursos para poner en práctica o el poco interés en hacerlo.

El conocimiento sobre los sistemas de cultivos ha evolucionado a lo largo del tiempo. En Junín, el conocimiento ecológico tradicional acerca de la preparación del suelo está basado en utilizar la tierra en determinados periodos, luego del cual debe "descansar" para mejorar su productividad. Además, es importante la rotación (cada año se siembra un producto diferente) y la asociación de cultivos (sembrar una especie principal y otra que ayude a conservar el suelo). También para la preparación del suelo se realiza el "chacmeo", actividad que prepara y remueve el suelo "descansado", haciéndola apta para el cultivo; luego se continúa con el "deshierbado" que consiste en sacar la hierba que perjudica el crecimiento del sembrío; y finalmente se lleva a cabo el "aporque", acumulado de tierra alrededor de la planta para favorecer su crecimiento. Estas prácticas son conductas ecoeficientes que ayudan el manejo óptimo de los suelos.

Se planteó como hipótesis de investigación: Existe relación significativa entre conocimiento ecológico tradicional y conductas hacia la ecoeficiencia agrícola en pobladores de la provincia de Junín. Para contrastarla, se aplicó la prueba de correlación tau b de Kendall con un nivel de significancia de 0.05. El coeficiente de correlación resultante fue $0.543(\mathrm{p}<0.05)$, que indica que a mayor conocimiento ecológico tradicional, mayor será la conducta hacia la ecoeficiencia agrícola (Tabla 3 ).

\section{Tabla 3}

Correlación entre prácticas conocimiento ecológico tradicional y conductas hacia la ecoeficiencia

\begin{tabular}{clc}
\hline & Tau b de Kendall & $\begin{array}{c}\text { Conductas hacia la } \\
\text { ecoeficiencia agrícola }\end{array}$ \\
\hline & Correlación & $\mathbf{0 . 5 4 3}^{* *}$ \\
Conocimiento & Sig. (bilateral) & $0.000<0.05$ \\
& $\mathrm{~N}$ & 210 \\
\hline
\end{tabular}

La correlación es significativa al nivel 0.01 (bilateral).

\section{DISCUSIÓN}

El conocimiento ecológico tradicional se relaciona significativamente con las conductas de ecoeficiencia agrícola de los pobladores de los pueblos originarios de la provincia de Junín, Perú, lo que significa que cuanto más conocimiento ecológico tradicional se tenga, mejor ecoeficiencia se logra tener. En este sentido, el enfoque científico positivista ha criticado duramente al enfoque tradicional de conservación biológica, pues la falta de consideraciones sociales disminuye su impacto y efectividad. También, la falta de marcos ontológicos y epistemológicos en los estudios del conocimiento ecológico tradicional es punto de quiebre que le quita el respaldo científico y su difusión en el mismo campo (Zalles, 2017). A pesar de ello, la conservación biológica, entendida desde las ciencias naturales, sigue siendo un elemento importante de conservación y desarrollo (Garcia et al., 2019; Zalles, 2017) 
Mediante el conocimiento ecológico tradicional, las personas se interrelacionan en las prácticas que pueden partir desde hacer un huerto familiar hasta hacer sembríos a gran escala, pues se comparten actividades socioculturales, lo que puede llevar a salvaguardar costumbres, tradiciones, creencias, ideologías y estructuras sociales en un determinado territorio (Castañeda - Guerrero et al., 2020; Garcia et al., 2019; Torres-Méndez, 2019). La crítica a los bancos genéticos como medio de solucionar problemas alimentarios de la población y conservar del equilibrio ecológico es sustentada y remarcada (Garcia et al., 2019). Desde otra perspectiva, Millaleo (2020) refuerza la importancia del conocimiento ecológico tradicional e incluso le da el enfoque legal, concluyendo a las cosmovisiones de los pueblos indígenas, quienes desarrollan la ética del cuidado, las diferentes relaciones de interconectividad y reciprocidad que forman el equilibrio socio ambiental.

Los diferentes agroecosistemas desarrollados con interacciones ecológicas están asociados al uso y manejo cultural de especies, que con el tiempo, desarrolla reservorios etnobiológicos, demostrando que el conocimiento ecológico tradicional favorece al desarrollo sostenible de los pueblos (Castañeda - Guerrero etal.,2019).

Torres-Méndez (2019) concluyó que si se perdiera el conocimiento tradicional no solo habría un quebrantamiento conceptual, sino que también se perdería cultura, técnicas e identidad. Aquí es importante resaltar que ni el gobierno de México ni el de Perú tienen estudios específicos sobre el conocimiento tradicional y de la trascendencia en la vida de los antiguos y nuevos pobladores, así como en la pervivencia de los demás seres vivos. Así, el conocimiento ecológico tradicional de los pueblos no es comprendido por las sociedades modernas, pues restan importancia a la armonía que conserva con la "Papatūānuku" (Nueva Zelanda) o "Pachamama" (Perú); sin embargo, esta simbiosis entre ser humano, naturaleza y Madre Tierra, no solo practica y comprende en un amplio espectro las diversas especies de plantas y animales, sino que la interrelación se desarrolla en una continua gratitud y respeto a la madre tierra (Huambachano, 2019).

Todas estas investigaciones han tenido como hallazgo principal que el conocimiento ecológico tradicional no solo conserva los diferentes ecosistemas y especies, sino que también permite cubrir las necesidades alimentarias de las personas, sin romper el equilibrio de la biomasa y asegurando más oportunidades de subsistencia a las generaciones futuras.

\section{CONCLUSIONES}

Los resultados muestran que los pobladores poseen notable conocimiento tradicional y conductas ecoeficientes en el tratamiento del suelo y en el proceso productivo. Su conocimiento y actitudes ecoeficientes en mantenimiento y gestión del agua es notable. Sin embargo, poseen un bajo conocimiento tradicional y actitudes ecológicas frente al tratamiento de plagas. Esto es un indicador de las limitaciones con las que los pobladores enfrentan estos inconvenientes y el potencial riesgo de pérdida de productos antes de la cosecha.

\section{REFERENCIAS}

Achkar, M., Canton, V., Cayssials, R., Domínguez, A., Fernández, G., \& Pesce, F. (2005). Indicadores de sustentabilidad. Comisión Sectorial de Educación Permanente. DIRAC, Facultad de Ciencias. Montevideo.

Alpuche, A. (2019). La reserva biocultural del Puuc en Yucatán. Desafíos de un nuevo modelo para la gestión del patrimonio cultural y natural. México: Plumas negras editorial.

Álvarez, H. (2017). Reinventar la educación desde la ecología social y los saberes ancestrales rurales. Universidad Simón Bolívar. Colombia.

Arnaiz-Schmitz, C., Herrero-Jáuregui, C., \& Schmitz, M. (2018). Losing a heritage hedgerow landscape. Bioculturaldiversity conservation in a changing social-ecological. Mediterranean system. Science of the Total Environment 637-638, 374-384. https://doi.org/10.1016/j.scitotenv.2018.04.413 
BDPI. (2020). Base de Datos de los Pueblos Indígenas u originarios. Ministerio de Cultura. Gobierno del Perú. Disponible en: https://bdpi.cultura.gob.pe/busqueda-de-localidades-de-pueblos-indigenas

Barcia-Piedras, J. (2020). Fitodesalinización asistida por microorganismos para la recuperación de suelos agrícolas (Doctoral dissertation). Universidad de Sevilla.

Bolaños, F. (2016). Dimensiones del problema ambiental contemporáneo. Disponible en: http://sgpwe.izt.uam.mx/files/users/uami/ifig/DIMENSIONES_DEL_PROBLEMA_AMBIENTA L_CONTEMPORANEO_Articulo.pdf

Castañeda-Guerrero, I., Aliphat-Fernández, M., Caso-Barrera, L., Lira Saade, R., \& Martínez Carrera, D. (2020). Conocimiento tradicional y composición de los huertos familiares totonacas de Caxhuacan, Puebla, México. Polibotánica, (49), 185-217.https://doi.org/10.18387/polibotanica.49.13

Castillo, S., \& Venegas, Y. (2016). Saberes ancestrales y prácticas productivas del pueblo Pumé como premisas de sustentabilidad agroecológica. Novum Scientiarum, 1(2), 25-36.

De la Riva, M. (2019). Conocimiento tradicional como estrategia de adaptación al Cambio Climático. Estudios de caso altiplano y valle interandino (Bolivia). Visiones sobre el clima y gestión del riesgo climático. Estudios y propuestas de estrategias de adaptación al Cambio Climático. Bolivia.

Fonseca, V. (2016) Las historias de la chagra: transformaciones en el conocimiento ecológico tradicionaly su influencia sobre los sistemas productivos del resguardo indigena. El Vergel, Amazonas.

García, J., Gutiérrez, J., Balderas, M., \& Pérez, J. (2019). Análisis del conocimiento ecológico tradicional y factores socioculturales sobre huertos familiares en el Altiplano Central Mexicano. Cuadernos Geográficos. 58. https://doi.org/10.30827/cuadgeo.v58i3.7867.

Gilles, J., \& Yucra, E. (2019). Vinculando conocimientos locales y cientificos: el caso de manejo de riesgos climáticos.

Huambachano, M. (2019). Soberanía alimentaria indígena: Recuperando el alimento como medicina sagrada en Aotearoa Nueva Zelanda y Perú. New Zealand Journal of Ecology, 43(3), 3383-ES

Ikeda, D. (2020). La construcción de una era de solidaridad humana: Hacia un futuro para todos.

Levy, S., Román, F., Douterlungne, D., Aguirre, J., \& Pérez, S. (2015). Conocimiento ecológico tradicional maya y rehabilitación de selvas.

Lima-da Silva, T., de Almeida-Campos, J., Chaves-Alves, Â., \& Albuquerque, U. (2019). Market integration does not affecttraditional ecological knowledge but contributes additional pressure on plant resources. Acta Botanica Brasilica, 33, 1-9. http://doi.org/10.1590/0102-33062018abb0310

Millán-Rojas, L., Arteaga-Reyes, T., Moctezuma-Pérez, S., Velasco-Orozco, J. \& Arzate-Salvador, J. (2016). Conocimiento ecológico tradicional de la biodiversidad de bosques en una comunidad matlatzinca, México. Ambiente y Desarrollo, 20(38), $111-123$. http://dx.doi.org/10.11144/Javeriana.ayd20-38.cetb

Millaleo, S. (2020). Guarda de la Naturaleza: Conocimientos Ecológicos Tradicionales de los Pueblos Indígenas y Estrategias de Protección. Universidad de Chile. Cadernos de Dereito Actual, 13, 202230.

Riechmann, J. (1995). Desarrollo sostenible: la lucha por la interpretación, en VV.AA., De la economía a la ecología. Madrid: Editorial Trotta y Fundación $1^{\circ}$ de Mayo.

Rodríguez, E. (2018). Indicadores de Ecoeficiencia de sistemas productivos agrícolas de la Altillanura Plana en la Orinoquia Colombiana. Disponible en: https://repositorio.unal.edu.co/handle/unal/64772

Ruano, J., Benítez, F., \& Larrea, A. (2020). Educación ambiental y praxis intercultural desde la filosofía ancestral del Sumak Kawsay. Utopía y praxis latinoamericana: revista internacional de filosofía iberoamericana y teoría social, (90), 120-135.

Shackleton, C., Pandey, A., \& Ticktin, T. (2015). Ecological sustainability for non-timber forest products. New York: Routledge. 
Torres-Méndez, S., Caso-Barrera, L., \& Aliphat-Fernández, M. (2019). Conocimiento ecológico, alimentación tradicional y clasificación frío-caliente: la perspectiva de los niños tseltales de Tenejapa, Chiapas. LiminaR, 17(2), 148-166.https://doi.org/10.29043/liminar.v17i2.673

Twarog, S., \& Promila, K. (2004), Preserving, Protecting and Promoting Traditional Knowledge: National Actionsand International Dimensions.

Vargas, I. (2011) Sistemas de Conocimiento Ecológico Tradicional y sus Mecanismos de Transformación: El caso de una chagra Amazónica. Universidad Nacional de Colombia.

Zalles, J. (2017) Conocimiento ecológico local y conservación biológica: la ciencia postnormal como campo de interculturalidad. Íconos - Revista de Ciencias Sociales 21(59), 205 